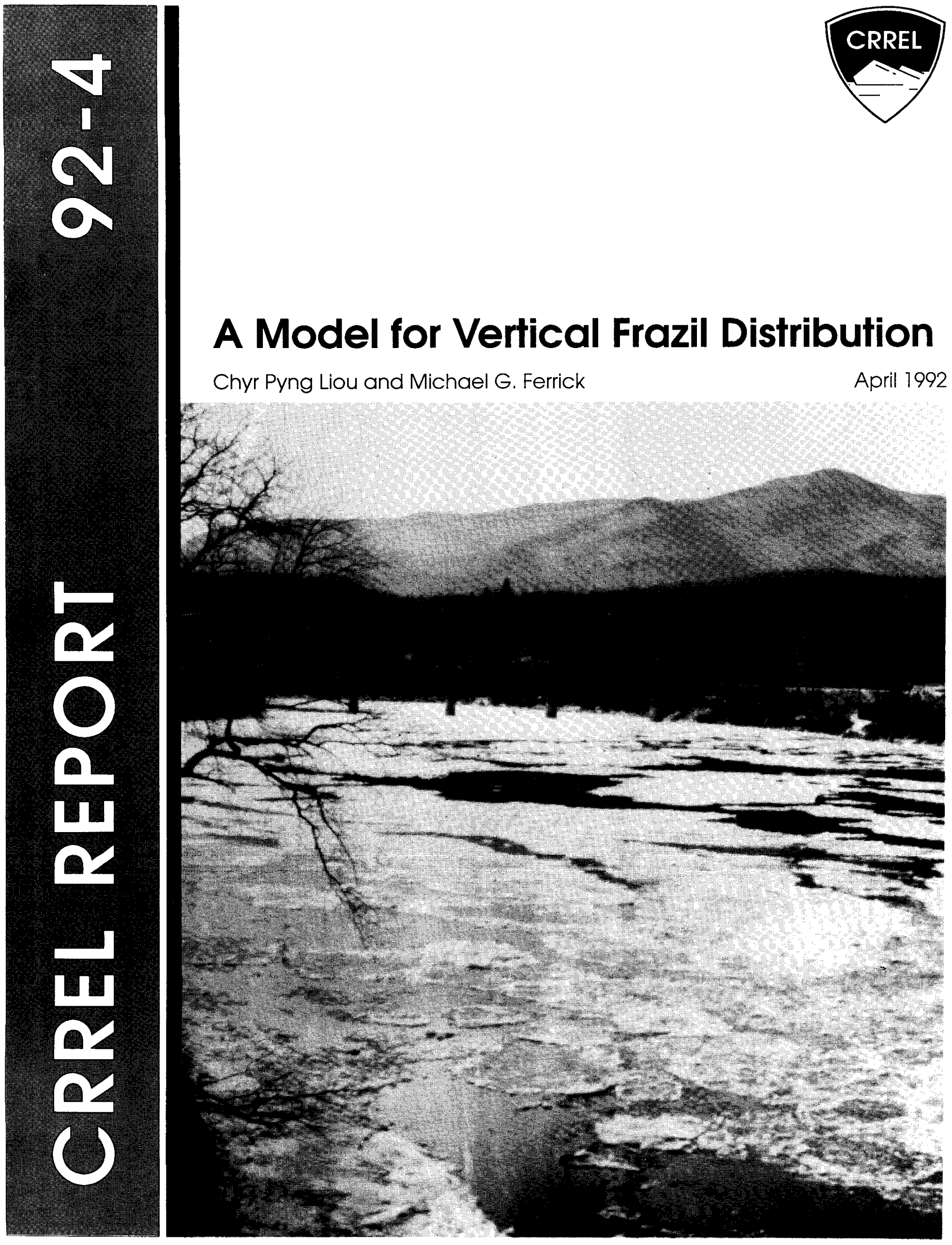


For conversion of SI metric units to U.S./British customary units of measurement consult ASTM Standard E380, Metric Practice Guide, published by the American Society for Testing and Materials, 1916 Race St., Philadelphia, Pa. 19103.

Cover: Layered frazil ice flow in the form of pans on the Connecticut River at Cornish, New HampshireWindsor, Vermont. (Photo by M. Ferrick) 


\section{CRREL Report 92-4}

U.S. Army Corps of Engineers

Cold Regions Research \& Engineering Laboratory

\section{A Model for Vertical Frazil Distribution}

Chyr Pyng Liou and Michael G. Ferrick 


\section{PREFACE}

This report was prepared by Chyr Pyng Liou, Department of Civil Engineering, University of Idaho, and Michael G. Ferrick, Research Hydrologist, Snow and Ice Branch, Research Division, U.S. Army Cold Regions Research and Engineering Laboratory. Funding for this work was provided by DA Project 4A161 102AT24, Research in Snow, Ice and Frozen Ground, Task SS, Work Unit 001, Snow and Ice Geophysics.

The authors thank Steven Daly and John Gagnon for help with the flume tests. They also thank Steven Daly, Mark Hopkins and Donald Perovich for their helpful reviews of the manuscript, Donna Harp for preparing many drafts, and Edmund Wright for careful editing.

The contents of this report are not to be used for advertising or promotional purposes. Citation of brand names does not constitute an official endorsement or approval of the use of such commercial products. 


\section{NOMENCLATURE}

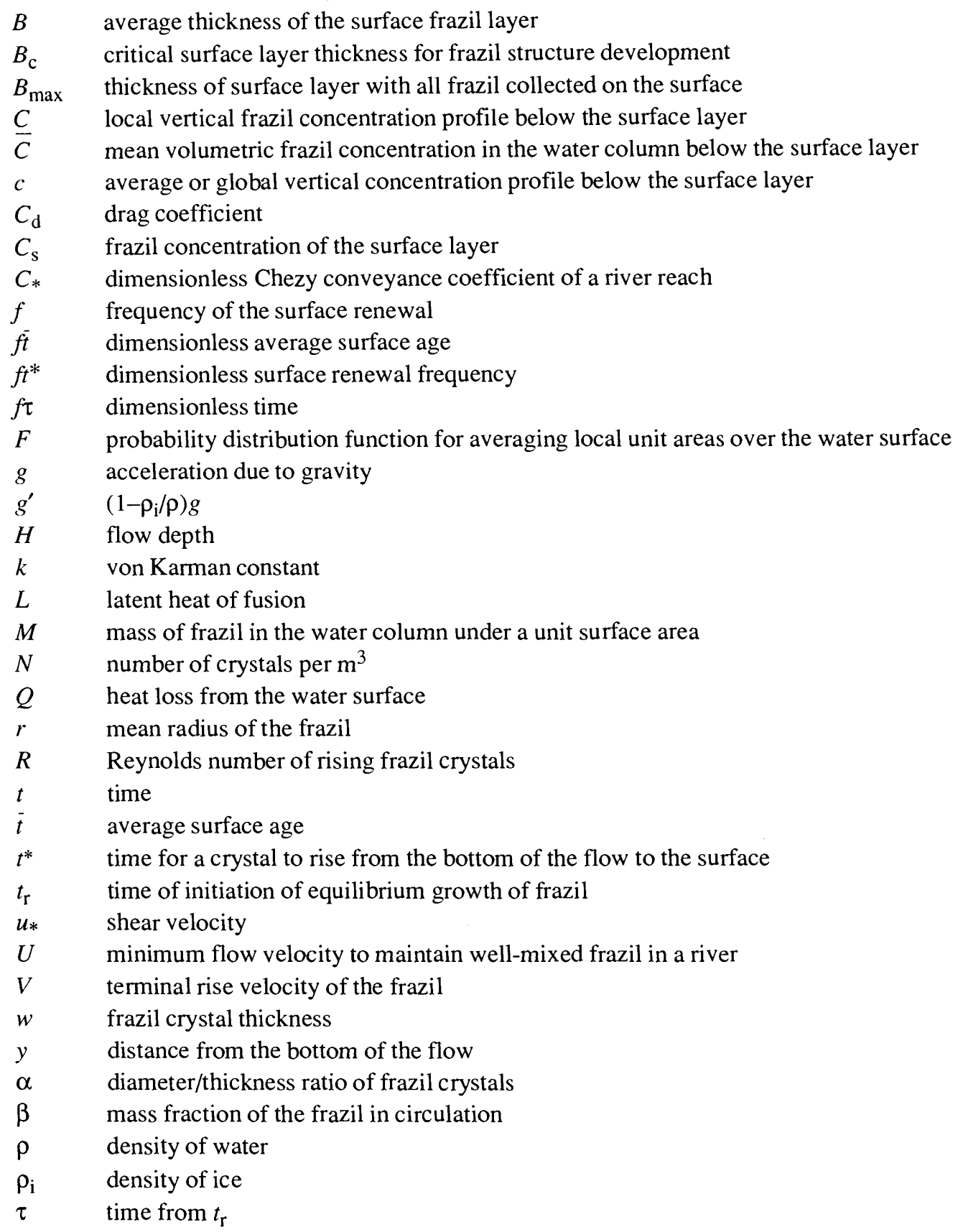




\section{CONTENTS}

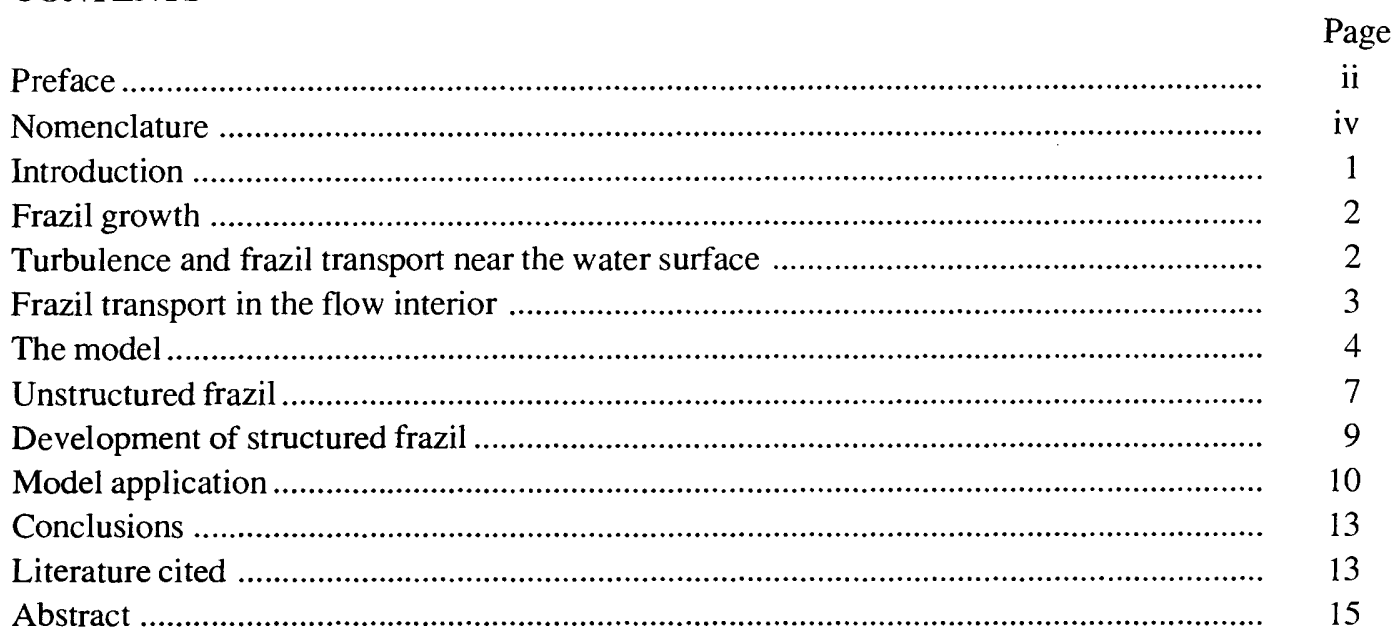

\section{ILLUSTRATIONS}

Figure

1. Rise velocity of frazil crystals .....................................................................................

2. Dimensionless average surface age as a function of dimensionless time ................... 5

3. Local frazil concentration profile ............................................................................. 5

4. Relative frazil concentration $c(y, \tau) / \bar{C}(\tau)$ profiles through time $\tau / t^{*}$ for selected

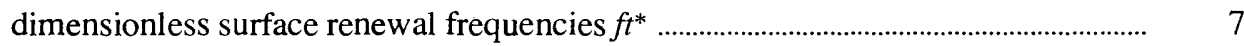

5. Equilibrium relative frazil concentration $c(y, \tau) / \bar{C}(\tau)$ profiles as a function of dimensionless surface renewal frequency $f t^{*}$................................................................

6. Nondimensional equilibrium surface layer thickness as a function of dimensionless surface renewal frequency.

7. Dimensionless surface layer thickness as a function of dimensionless time for selected dimensionless critical surface layer thicknesses and surface renewal frequencies

8. $\beta$ as a function of dimensionless time for selected dimensionless critical layer thicknesses and surface renewal frequencies

9. $\beta$ and $B / B_{\max }$ as functions of normalized dimensionless time for selected values of $f t^{*}$ and $B_{\mathrm{c}} / B_{\max }$

10. Normalized frazil concentration as a function of dimensionless depth and normalized dimensionless time for selected values of $f t^{*}$ and $B_{\mathrm{c}} / B_{\max }$ 


\title{
A Model for Vertical Frazil Distribution
}

\author{
CHYR PYNG LIOU AND MICHAEL G. FERRICK
}

\section{INTRODUCTION}

Frazilice consists of individual disk-shaped crystals formed in turbulent water. It is initiated by seeding at the air/water interface, followed by secondary nucleation (Osterkamp 1978, Osterkamp et al. 1983, Daly 1984, Ashton 1986). Wide ranges of frazil disk diameter and thickness have been reported (Schaefer 1950, Arakawa 1954, Kivisild 1970, Daly and Colbeck 1986). Due to the higher growth rate in the basal plane of an ice crystal (Hobbs 1974), young frazil crystals are smaller and thicker than older ones. Frazil crystal concentrations in natural rivers range from 10 thousand to 1 million crystals per cubic meter (Schaefer 1950, Osterkamp and Gosink 1982). If we assume a diameter/thickness ratio of 30 , the volume concentration corresponding to 1 million 3-mm-diam. crystals per cubic meter is 0.0007 ; thus the frazil-water mixture is very dilute. Frazil crystals that are actively growing in slightly supercooled water can attach to any solid surface upon contact, and can lead to ice dams, anchor ice, and icing of water intake structures in rivers. Frazil is also believed to be an important mode of ice growth in the marginal ice zones of the Arctic and the Antarctic. Osterkamp (1978), Martin (1981), Daly (1984) and Ashton (1986) review frazil formation and growth.

The evolution of frazil into larger surface ice forms such as flocs, pancakes, and eventually floes is not completely understood. Michel (1978) proposed that frazil crystals agglomerate to form porous flocs within the flow during the active growth stage, and then concentrate at the water surface as frazil slush. If the slush is dense and stays at the surface long enough, ice floes in the shape of pancakes develop. Larger surface ice forms may evolve from these pancakes. However, since the frazil-water mixture is very dilute, the chance for crystals to collide and form flocs in the flow interior is low relative to that at the surface, and has not been well documented.

Because they are buoyant, frazil crystals tend to concentrate near the water surface, increasing the opportunities for collisions between crystals. The crystals at the water surface in direct contact with subfreezing air have a greater potential for heat loss and freeze bonding than those at depth. Therefore, structured frazil accumulations are more likely to develop at the water surface than in the interior of the flow. Martin and Kauffman (1981) found from their saltwater wave tank experiment that frazil crystals floated to the water surface and were herded by the waves to one end of the tank. The individual crystals rapidly sintered together to form large irregular clumps. Osterkamp and Gosink (1982) suggested that at high velocities, turbulence may prevent flocculation and ice pan formation, and instead, frazil slush may develop at the surface. If the turbulence is strong enough, frazil may be kept in suspension and the water surface may be essentially free of ice. Hirayama (1986) reported field observations of both suspended and layered frazil. Tsang (1988) proposed a model for frazil distribution based on energy considerations, and compared the results with limited field data.

In this report we consider the processes in a turbulent flow that determine if frazil crystals will remain in suspension or if more structured ice will form at the water surface. We propose a model to enhance our understanding of the physical processes involved in frazil evolution, to systematically assess observations of mixed and layered flows, and to provide a logical structure to guide further work. 


\section{FRAZIL GROWTH}

Frazil grows through the removal of the heat of fusion by turbulence and the eventual heat transfer to the air above the water surface. Carstens (1966) and Osterkamp (1978) present an idealized water temperature history during periods of frazil production and growth. When the air temperature is subfreezing, the water temperature can drop below the freezing point. Frazil nucleation begins soon after, and the rate of temperature decrease slows as latent heat is released by the growing crystals. The water temperature then reaches a minimum and begins to recover as the rate of ice growth increases. Eventually an equilibrium is reached at time $t_{\mathrm{r}}$ when the rate of heat released by the crystals equals the rate of heat loss to the air. The dynamics of initial frazil formation and growth are not well understood. The modes of ice growth that can occur are crystal number growth and crystal size growth. The initial period of nonequilibrium growth prior to $t_{\mathrm{r}}$ is believed to be brief (minutes). After $t_{\mathrm{r}}$, a slight amount of supercooling in the water is maintained, which is responsible for the continued growth of frazil crystals. Detailed models of nonequilibrium frazil growth can be found in Daly (1984) and Omstedt (1985).

During the equilibrium growth period after $t_{\mathrm{r}}$, the time rate of increase of frazil mass $M$ in the water column under a unit surface area can be related to the rate of heat loss $Q$ from the surface by the principle of energy conservation:

$$
L \frac{d M(\tau)}{d \tau}=Q(\tau)
$$

where $L$ is the latent heat of fusion and $\tau$ denotes time since $t_{\mathrm{r}}$. Equation 1 is integrated to obtain the total mass of frazil in the water column at $\tau$ :

$$
M(\tau)=\frac{1}{L} \int_{0}^{\tau} Q(t) d t
$$

The integration constant can be determined using $M=M_{\mathrm{r}}$ at $\tau=0$. The ice mass increases with time as long as the surface heat loss continues.

\section{TURBULENCE AND FRAZIL TRANSPORT NEAR THE WATER SURFACE}

Large surface features such as boils and eddies, attributable to turbulence, are often visible. Komori et al. (1982) conducted a laboratory study on turbulence and transport mechanisms at the water surface in a shallow open-channel flow. They found that 1 ) the intensity of vertical velocity fluctuations is reduced near the free surface, which increases the intensity of velocity fluctuations in the streamwise and lateral directions, 2) large energy-containing eddies well up toward the surface, spread horizontally, then plunge back into the flow interior, and 3) the time fraction when these eddies are at the surface is only about $10 \%$, but they contribute more than $60 \%$ of the mixing near the surface. Similar turbulence structures with $60 \%$ of the Reynolds stress produced by large eddy motions occupying only $10 \%$ of the time were observed by Seitz (1973) in an estuary and by Gordon and Dohne (1973) in a river. Additional information on this subject can be found in Brown (1970), Davis and Lozano (1979), Brutsaert and Jirka (1984), Shimko (1985) and Sonin et al. (1986).

We have also observed large eddy motion during experiments in the $36-\mathrm{m}$-long and $1.2-\mathrm{m}$-wide tilting flume at the Cold Regions Research and Engineering Laboratory. A flow rate of $0.057 \mathrm{~m}^{3} / \mathrm{s}$ was used with both a bare aluminum channel floor and a roughened channel floor that increased the Manning's roughness of the flume by a factor of 10 . Talc powder was spread over the water surface near the upstream end of the flume, and this patch of the surface was videotaped with a camera mounted 
on an instrument carriage that moved with the flow. The upwelling of interior water to the surface was vivid as the eddy motions temporarily cleared the talc at various locations in a seemingly random paitern. The sizes of the renewed areas were of the order of the flow depth. This renewal behavior was L. $\quad$ I for all flow conditions except for the lowest velocity $(0.1 \mathrm{~m} / \mathrm{s})$ case in the smooth channel.

We propose that the surface renewal eddies act as the primary mechanism whereby frazil crystals at the water. face are entrained and redistributed over the depth of the turbulent flow. A large eddy a the wai urface that is uniformly covered with frazil crystals and momentarily clears an area. As tie eddy plunges back into the flow interior, it entrains some of the crystals that occupied the temporarily cleared area. We assume that the entrained crystals and the crystals in the flow interior are uniformly redistributed over the flow depth by the eddy mixing. Afterward, the net upward motion resumes. The large eddies also promote horizontal surface currents as they spread. The opposing surface currents between closely spaced eddies herd the frazil crystals into the narrow region between them. The concentrated crystals are exposed to subfreezing air and may agglomerate to form flocs.

The frequency $f$ of the surface renewal affects the time available for the frazil at the water surface to become more structured. We assume $f$ to be uniform over the water surface and constant over time. Methods of estimating $f$ can be found in the literature on gas absorption over turbulent liquid surfaces. O'Connor and Dobbins (1958) present an argument, based on mixing length theory, that $f$ equals the vertical velocity gradient at the surface. By assuming a logarithmic velocity distribution, they showed that

$$
f=\frac{u *}{k H}
$$

where $k=$ the von Karman constant

$H=$ the flow depth

$u_{*}=$ the shear velocity.

Komori et al. (1982) measured $f$ to be $0.82 \mathrm{~Hz}$, which expressed in terms of shear velocity and flow depth replaces $1 / k$ in eq 3 by 3.73 . We will use eq 3 with the experimentally determined constant to estimate $f$.

\section{FRAZIL TRANSPORT IN THE FLOW INTERIOR}

Between renewals, the rise velocity due to the buoyancy of frazil in the interior depends on size, shape and orientation of the crystals, and on the turbulence associated with eddies smaller than the energy-containing ones. Since frazil crystals have a range of sizes at any instant, a distribution of rise velocities exists. However, for simplicity of this first generation model, we make the assumption that the number of crystals is a constant and consider the mean radius $r$ of the frazil as a function of time:

$$
r(\tau)=\left(\frac{M(\tau) \alpha}{2 \pi N H \rho_{\mathrm{i}}}\right)^{1 / 3}
$$

and the frazil crystal thickness $w$ as a function of time:

$$
w(\tau)=\frac{2 r(\tau)}{\alpha}
$$

where $\alpha=$ a representative diameter/thickness ratio of the crystals

$\rho_{\mathrm{i}}=$ the density of ice

$N=$ the number of crystals per cubic meter, typically between $10^{3} / \mathrm{m}^{3}$ and $10^{6} / \mathrm{m}^{3}$. 
We assume that during the time between renewals the crystals rise at their terminal velocity: the velocity at which the buoyancy force balances the drag force. Wuebben (1984) observed that frazil crystals in a quiescent water column rise without rocking or tumbling in a favored orientation of basal plane normal to the vertical. Based on this force balance, and assuming the plane of the crystal disk to be horizontal, we can express the terminal velocity $V(\tau)$ as

$$
V(\tau)=\left[\frac{4 g^{\prime} r(\tau)}{\alpha C_{\mathrm{d}}}\right]^{1 / 2}
$$

where $g^{\prime}=g\left(1-\rho_{\mathrm{i}} / \rho\right)$

$g=$ acceleration due to gravity

$C_{\mathrm{d}}=$ the drag coefficient.

Gosink and Osterkamp (1983) obtained $C_{\mathrm{d}}$ by fitting observed data as

$$
\log C_{\mathrm{d}}=1.386-0.8921 \log R+0.111(\log R)^{2}
$$

where the Reynolds number $R$ is based on the rise velocity and the diameter of the frazil disk. They found that the rise velocity calculated from eq 6 and 7 , with diameter-to-thickness ratios of 10 and 50 , bracketed the observed values for disks with diameters between 1 and $3 \mathrm{~mm}$. These data, presented in Figure 1, included both quiescent laboratory water columns and agitated water columns in the field. All of these data were comparable. The scatter may be a result of the small eddies.

\section{THE MODEL}

Our model is based on several hypotheses. The scale of the energy-containing eddies is comparable to the depth of the shear flow. During a surface renewal the frazil is mixed downward, primarily by advection by the large eddies. We neglect the mixing caused by smaller eddies. Between surface renewals the buoyancy of the growing crystals dictate upward movement. The frazil crystals at the surface bond if the concentration is high and the time of exposure to subfreezing air is sufficiently long. The formation of crystal floes at the surface results in increased buoyant force and resistance to reentrainment by the large eddies.

The water surface is occasionally renewed at individual locations by the large eddies. We will not characterize the mixing action of these eddies individually. Instead we consider their gross effect on the frazil concentration distribution by averaging local unit areas over the entire surface with a probability distribution function (PDF). We

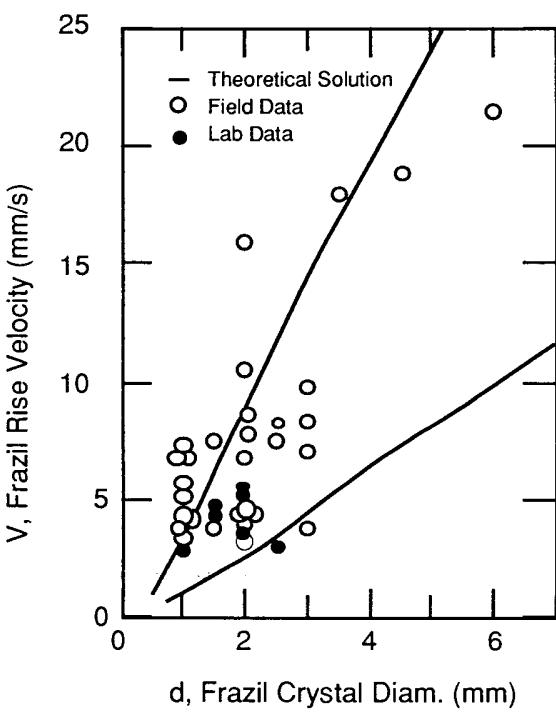

Figure 1. Rise velocity of frazil crystals (after Gosink and Osterkamp 1983).The upper and lower lines represent diameterl thickness ratios of 10 and 50, respectively.

$$
F(t)=\frac{f e^{-f t}}{1-e^{-f \tau}} .
$$




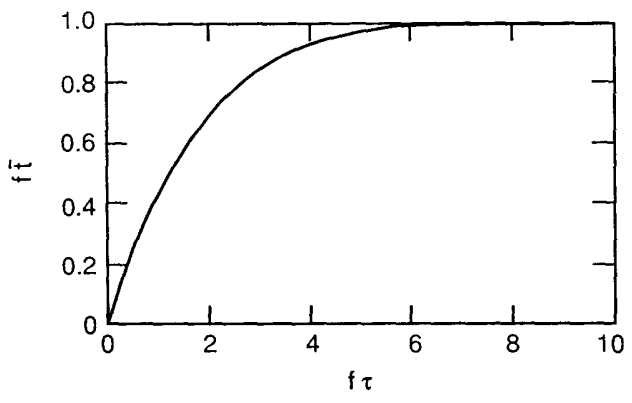

Figure 2.Dimensionlessaverage surface age as a function of dimensionless time.

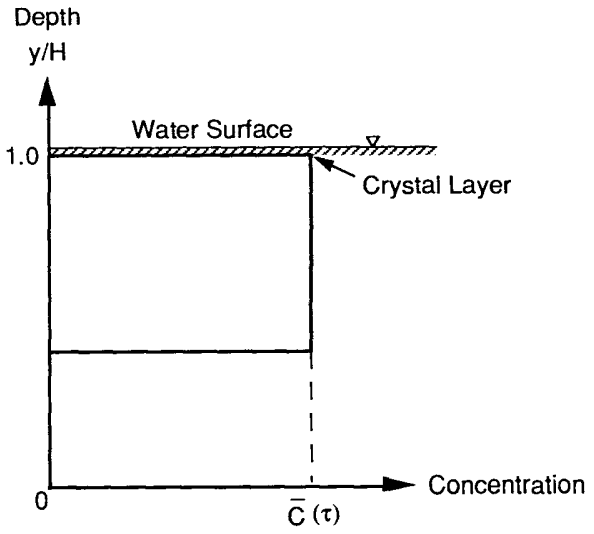

Figure 3. Local frazil concentration profile.

$F(t)$ is positive and for $0 \leq t \leq \tau$ has an integral of 1 , requirements for a PDF. Dankwerts (1951) proposed a similar PDF for modeling gas absorption across a free surface, and eq 8 approaches Dankwerts' PDF as $\tau$ becomes large. Komori (1982) experimentally demonstrated the utility of Dankwerts' PDF by establishing a mass transfer coefficient for the liquid. We chose the PDF given in eq 8 because the mixing of a surface layer into the interior of a flow is the same for both gas absorption and frazil development.

The residence time of frazil at the surface is important in the development of structure, and can be characterized by surface age. The age of a surface area is the time $t$ since the last renewal or since $t_{\mathrm{r}}$, whichever is shorter. At time $\tau$ the age of the water surface patches vary between 0 and $\tau$, and the fraction with ages between $t$ and $t+d t$ is $F(t) d t$. The average surface age $\bar{t}$ is

$$
\bar{t}=\int_{0}^{\tau} t F(t) d t
$$

or

$$
\overline{f t}=\frac{1-(1+f \tau) \mathrm{e}^{-f \tau}}{1-\mathrm{e}^{-f \tau}} .
$$

The dimensionless average surface age $f \bar{t}$ as a function of dimensionless time $f \tau$ obtained from eq 9 is presented in Figure 2.

If the rise velocity of frazil crystals in turbulent water is known, the time for a crystal at the bottom of the shear flow to rise to the water surface $t^{*}$ is

$$
t^{*}=\frac{H}{V(\tau)} .
$$

For values of $t$ less than $t^{*}$, the crystals move upward, leaving a space cleared of frazil near the bottom. Meanwhile, crystals accumulate in a surface layer of concentrated frazil. The local frazil concentration profile below the surface layer, shown in Figure 3, follows from the assumptions of uniform crystal size with a single rise velocity, and a uniform concentration after large eddy mixing. This profile is expressed as

$$
C(y, t)=\left\{\begin{array}{lll}
\bar{C}(\tau) & \text { for } & \frac{y}{H} \geq \frac{t}{t^{*}} \\
0 & \text { for } & \frac{y}{H}<\frac{t}{t^{*}}
\end{array}\right.
$$


where $\bar{C}(\tau)$ is the mean volumetric concentration of frazil in the water column. $\bar{C}(\tau)$ depends on the rate of heat loss at the surface $Q$ and time $\tau$. For $t>t^{*}$ all crystals are gathered in the surface layer.

Frazil crystals that gather at the water surface and are undisturbed for a sufficient period of time may become bonded and resist reentrainment into the flow interior by turbulent eddies. The mean volumetric concentration of frazil in the water column after $t_{\mathrm{r}}$ can be expressed as

$$
\bar{C}(\tau)=\beta(\tau) \frac{M(\tau)}{\rho_{\mathrm{i}} H}
$$

where $\beta(\tau)$ represents the mass fraction of frazil in circulation relative to the total mass of frazil. Initially all the frazil crystals are in circulation and $\beta(0)=1$. Subsequently $\beta(\tau)$ may decrease if structured frazil forms at the surface and resists reentrainment.

Using $F(t) d t$ as the weighting factor, we obtain an average or global vertical concentration profile $c(y, \tau)$ from the individual profiles under areas of different age as

$$
c(y, \tau)=\int_{0}^{\tau} C(y, t) F(t) d t
$$

With the average concentration profile we can establish the average thickness of the corresponding surface frazil layer $B(\tau)$. A balance equation written for the total ice volume on a unit surface area and in the water column below it at time $\tau$ yields

$$
B(\tau)=\frac{M(\tau)-\rho_{\mathrm{i}} \int_{0}^{H} c(y, \tau) d y}{\rho_{\mathrm{i}} C_{\mathrm{S}}}
$$

where $C_{\mathrm{s}}$ is the frazil concentration of the surface layer. Martin and Kauffman (1981) obtained the range of $C_{\mathrm{s}}$ as $0.2-0.4$. Our field observations of frazil in rivers indicate a lower $C_{\mathrm{s}}$ value. Data from Tsang (1988) indicate a $C_{\mathrm{s}}$ of 0.03 in a river. The maximum thickness of the surface layer can be obtained from eq 14 as

$$
B_{\max }=\frac{M(\tau)}{\rho_{\mathrm{i}} C_{\mathrm{s}}}
$$

We visualize a critical surface layer thickness as a threshold for the ice at the surface to develop structure. This critical thickness $B_{\mathrm{c}}$ depends on the freeboard of crystals at the surface and other environmental parameters. When the layer thickness exceeds $B_{c}$ the ice protrudes above the water surface and has remained there for a sufficient time to promote the bonding of crystals. These ice flocs have increased resistance to reentrainment and $\beta(\tau)<1$. Before this thickness is attained, all crystals on the surface are entrained back into the interior when the surface is renewed and $\beta(\tau)=1$. We assume that $\beta(\tau)$ is a function of the average surface layer thickness and the average surface age, and propose a simple expression that is correct at both extremes of time as

$$
\beta(\tau)=1-\frac{\bar{B}(\tau)}{B_{\max }-B_{\mathrm{c}}} f \bar{t}
$$

where

$$
\bar{B}(\tau)=\left(\begin{array}{lll}
0 & \text { for } & B(\tau)<B_{\mathrm{c}} \\
B(\tau)-B_{\mathrm{c}} & \text { for } & B(\tau) \geq B_{\mathrm{c}}
\end{array}\right.
$$

The development of a surface layer will reduce the rate of heat loss $Q(\tau)$ from the turbulent water 
column and inhibit the growth of the frazil crystals in circulation. When $Q(\tau)=0$, crystal growth stops, and the rise velocity ceases to increase.

\section{UNSTRUCTURED FRAZIL}

We can examine some aspects of the conceptual model using the idealized local concentration profile shown in Figure 3. Substituting eq 8 and 11 into 13 and performing the integration, we obtain

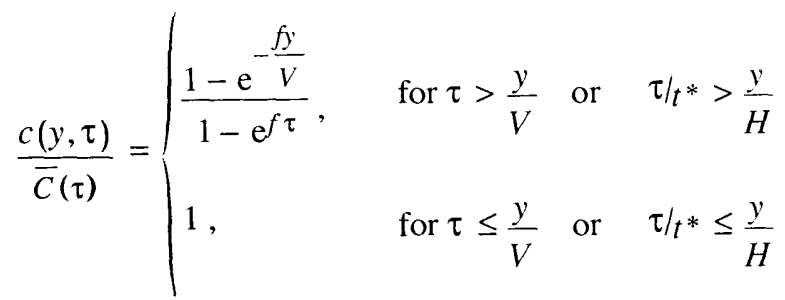

where $y / V$ is the time needed for a crystal to rise from the bottom to $y$. Given a specifc depth and time the relative concentration $c(y, \tau) / \bar{C}(\tau)$ depends on the ratio of the renewal frequency to the rise velocity. We use a dimensionless renewal frequency $\mathrm{ft}^{*}$ to characterize the relative magnitude of the downward motion of frazil by the renewal eddies with that of the upward motion by buoyancy of the crystals. Figure 4 shows the mean concentration profiles $c(y, \tau) /$ $\bar{C}(\tau)$ through dimensionless depth at dimensionless renewal frequencies of $0.1,1$ and 10 , and several values of dimensionless time $\tau / t^{*}$. We note in Figure 4 that at small dimensionless time the mean concentration is $\bar{C}(\tau)$ everywhere in the profile except near the bottom of the shear flow. At $\tau / t^{*}=1$ crystals originally at the bottom arrive at the surface. If a local renewal has not occurred, all the crystals reside at the surface. The average concentration profiles at $\tau / t^{*}=1$ range from zero at the bottom to $\bar{C}(\tau)$ immediately below the surface layer.

At larger dimensionless times and after the surface heat loss has ceased, the rate of frazil entrainment by the large eddies is balanced by buoyancy and a dynamic equilibrium develops. A portion of the frazil remains in suspension indefinitely beneath a surface layer of constant thickness that is without structure. The equilibrium frazil concentration profile at large $\tau$ is obtained from eq 17:

$$
\frac{c(y)}{\bar{C}(\tau)}=\left(1-\mathrm{e}^{-f y / V)}=\left(1-\mathrm{e}^{-f t^{*} y / H}\right)\right.
$$

The equilibrium profile with dimensionless depth is only a function of $f t^{*}$, and several profiles are given in Figure 5. When the dimensionless renewal frequency is large, the equilibrium profile reflects well-mixed conditions; when $f t^{*}$ is small, the frazil collects in a layer at the water surface. Note that if the surface heat loss persists longer, the mean crystal size increases and $f t^{*}$ becomes smaller. Then, with all other conditions the same, the resulting
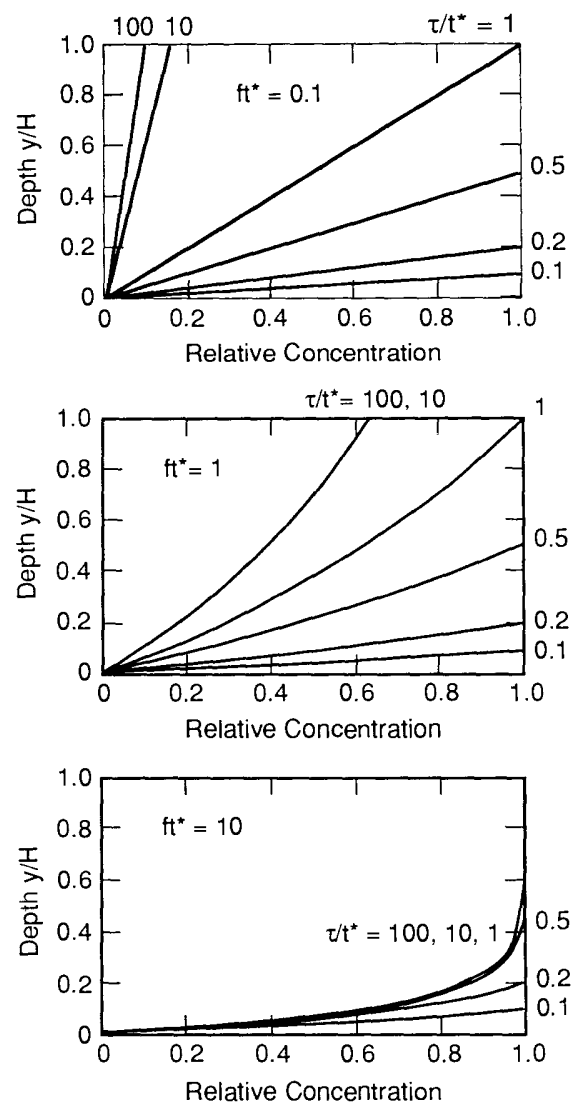

Figure 4. Relative frazil concentration $c(y, \tau) / \overline{\mathrm{C}}(\tau)$ profiles through time $\tau / \mathrm{t}$ for selected dimensionless surface renewal frequencies $\mathrm{ft}^{*}$. 


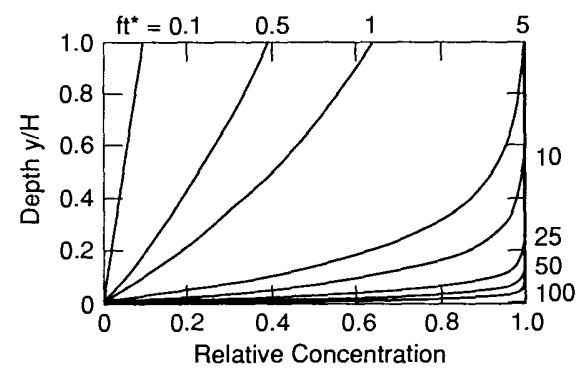

Figure 5. Equilibrium relative frazil concentration $\mathrm{c}(\mathrm{y}) / \overline{\mathrm{C}}(\tau)$ profiles as a function of dimensionless surface renewal frequency $\mathrm{ft}^{*}$.

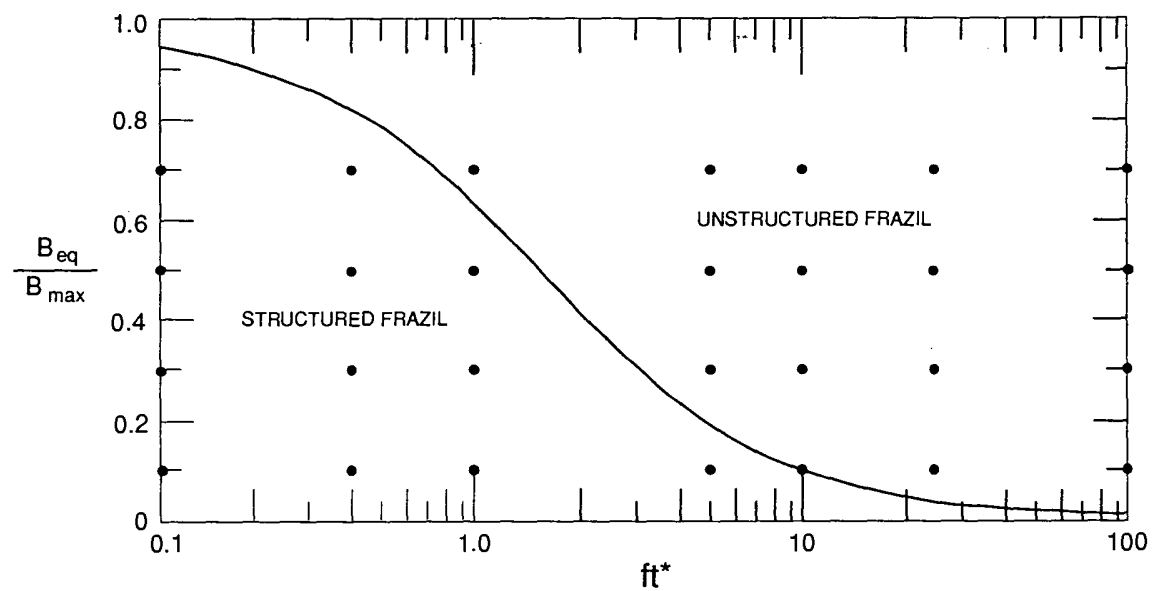

Figure 6. Nondimensional equilibrium surface layer thickness as a function of dimensionless surface renewal frequency. The curve defines regions of structured and unstructured frazil. The points shown correspond to the cases presented in Figures 8 and 9.

equilibrium state would have generally lower relative concentrations throughout the depth and much more frazil in the surface layer.

An equilibrium surface layer corresponds to each equilibrium profile. Substituting eq 18 into eq 14 , performing the integration, and rewriting using eq 12 with $\beta=1$ and eq 15 yields the equilibrium thickness of the surface layer $B_{\mathrm{eq}}$ as

$$
\frac{B_{\mathrm{eq}}}{B_{\max }}=\left(\frac{1-e^{-f t^{*}}}{f t^{*}}\right)
$$

The dimensionless equilibrium surface layer thickness obtained from eq 19 is given in Figure 6. Equilibrium thickness approaches $B_{\max }$ as $f t^{*}$ approaches zero, and it approaches zero as $f t^{*}$ becomes large. Thus, without bonding between the crystals, the surface layer remains unstructured, with an average thickness that is inversely related to $f t^{*}$. When $f t^{*}$ is sufficiently small, local patches of crystals at the surface become thick and have a long surface residence time. These conditions promote bonding between crystals, and subsequent renewal eddies may not entrain all the frazil on the patch of surface. The partial entrainment that results is accounted for in the model by $\beta(\tau)<1$. Bonding initiates the 
formation of ice flocs on the surface and a continual loss of crystals to the surface layer that will eventually deplete the flow interior.

\section{DEVELOPMENT OF STRUCTURED FRAZIL}

Structured frazil begins to develop at the water surface when $B(\tau)$ exceeds the threshold represented by $B_{\mathrm{c}}$. Beyond this threshold the equilibrium thickness no longer limits the layer thickness, which will approach $B_{\max }$ with time. The critical surface layer thickness made nondimensional by $B_{\max }$ can be compared with the dimensionless equilibrium thickness for the same $f t^{*}$. To allow the possibility of structured frazil, the equilibrium surface layer thickness must exceed $B_{\mathrm{c}}$. Therefore, the relative values of equilibrium and critical layer thickness define the regions of structured and unstructured frazil shown in Figure 6.

We obtain a set of equations for the dimensionless surface layer thickness $B(\tau) / B_{\max }$ using eq 8,11 , $12,13,14$ and 17 as

$$
\begin{aligned}
& \frac{B(\tau)}{B_{\max }}=\frac{1-(1+f \tau) \mathrm{e}^{-f \tau}}{f t^{*}\left(1-\mathrm{e}^{-f \tau}\right)} \quad, \quad \text { for } \frac{\tau}{t^{*}} \leq 1 \text { and } B \leq B_{\mathrm{c}} \\
& \frac{B(\tau)}{B_{\max }}=\frac{1-\left(\mathrm{e}^{-f t^{*}}+f t^{*} \mathrm{e}^{-f \tau}\right)}{f t^{*}\left(1-\mathrm{e}^{-f \tau}\right)} \quad, \quad \text { for } \frac{\tau}{t^{*}}>1 \text { and } B \leq B_{\mathrm{c}} \\
& \frac{B(\tau)}{B_{\max }}=\frac{1-\mathrm{e}^{-f \tau}(1+f \tau)+\left(\frac{B_{\mathrm{c}} / B_{\max }}{1-B_{\mathrm{c}^{\prime}} B_{\max }} f^{-}\right)\left[1-f t^{*}-\mathrm{e}^{-f \tau}\left(1+f \tau-f t^{*}\right)\right]}{f t^{*}\left(1-\mathrm{e}^{-f \tau}\right)\left(\frac{1}{1-B_{\mathrm{c}} B_{\max }} f \bar{t}\right)\left[1-f t^{*}-\mathrm{e}^{-f \tau}\left(1+f \tau-f t^{*}\right)\right]}
\end{aligned}
$$

$$
\begin{aligned}
& \text { for } \frac{\tau}{t^{*}} \leq 1 \quad \text { and } \quad B>B_{\mathrm{c}}, \quad \text { and } \\
& \frac{B(\tau)}{B_{\max }}=\frac{1-\left(\mathrm{e}^{-f t^{*}}+f t^{*} \mathrm{e}^{-f \tau}\right)+\left(\frac{B_{\mathrm{c}} / B_{\max }}{1-B_{\mathrm{c}} B_{\max }} f \bar{t}\right)\left[1-f t^{*}-\mathrm{e}^{\left.-f t^{*}\right]}\right.}{f t^{*}\left(1-\mathrm{e}^{-f \tau}\right)+\left(\frac{1}{1-B_{\mathrm{c}} / B_{\max }}-\bar{t}\right)\left[1-f t^{*}-\mathrm{e}^{\left.-f t^{*}\right]}\right.} \\
& \text { for } \frac{\tau}{t^{*}}>1 \quad \text { and } B>B_{\mathrm{c}}
\end{aligned}
$$

The dimensionless surface layer thickness as a function of dimensionless time is given in Figure 7 and the mass fraction of frazil in circulation $\beta$ as a function of dimensionless time is given in Figure 8 for ranges of $f t^{*}$ and $B_{\mathrm{c}} / B_{\max }$. With $B_{\mathrm{c}} / B_{\max }=0.1$ and $f t^{*} \geq 10$ the surface layer remains unstructured with a dynamic equilibrium between the frazil on the surface and that in the interior. For these conditions $\beta$ is constant with a value of 1 . The surface layer thickness eventually exceeds $B_{\mathrm{c}}$ if $f t^{*}$ becomes less than 10 , and in time the flow interior is depleted of crystals. $B / B_{\max }$ approaching 1 and $\beta$ approaching zero indicate this condition. Larger $B_{\mathrm{c}} / B_{\max }$ values allow a mixed flow dynamic equilibrium at smaller dimensionless renewal frequencies. The final surface layer thickness can only be $B_{\max }$ with $\beta=0$, or $B_{\text {eq }}$ with $\beta=1$. 

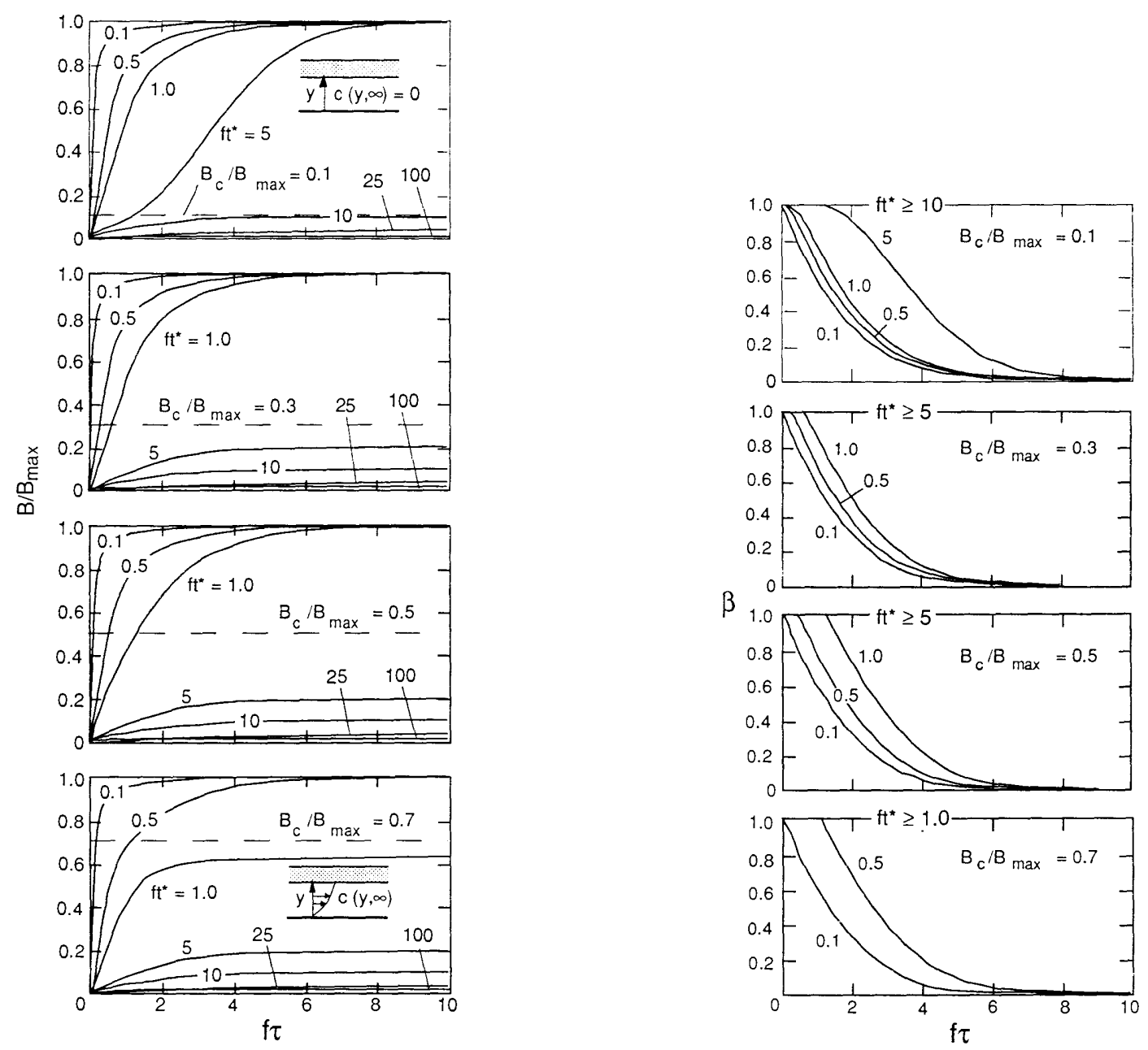

Figure 7. Dimensionless surface layer thickness as a function of dimensionless time for selected dimensionless critical surface layer thicknesses and surface

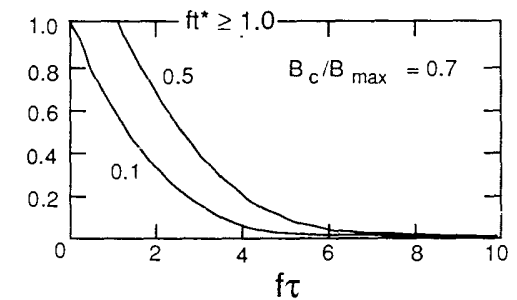

Figure 8. Bas afunction of dimensionless time for selected dimensionless critical layer thicknesses and surface renewal frequencies.

\section{MODEL APPLICATION}

We now compare the model with empirical relationships and field observations that have been reported in the literature. Matousek (1984) proposed a formula for the critical velocity $U$ (in $\mathrm{m} / \mathrm{s}$ ) required to maintain well-mixed frazil in a river:

$$
U \geq \frac{5 V}{g^{1 / 2}}[C(0.7 C+6)]^{1 / 2}
$$

where $C=g^{1 / 2} C_{*}$ is the Chezy coefficient and $C *$ is the dimensionless Chezy coefficient. The critical velocity is related to the shear velocity as $U=u_{*} C_{*}$. Using eq $21, f=3.73 u_{*} / H$, and a constant $V$ of $0.01 \mathrm{~m} / \mathrm{s}$ with $C_{*}$ values of 5 and 30 yields dimensionless renewal frequencies of 19 and 16 , respectively. The empirical relationship of Gosink and Osterkamp (1983) for approximating the velocity threshold between layered and well-mixed frazil is $U=C / 42$, or in terms of shear velocity $u *$ $=0.075 \mathrm{~m} / \mathrm{s}$. Again, assuming $V=0.01 \mathrm{~m} / \mathrm{s}$ the corresponding $f t^{*}=28$. The equilibrium profiles in 
Figure 9. $\beta$ and $\mathrm{B} / \mathrm{B}_{\max }$ as functions of normalized dimensionless time for selected values of $\mathrm{ft}^{*}$ and $\mathrm{B}_{c} / \mathrm{B}_{\max }$.
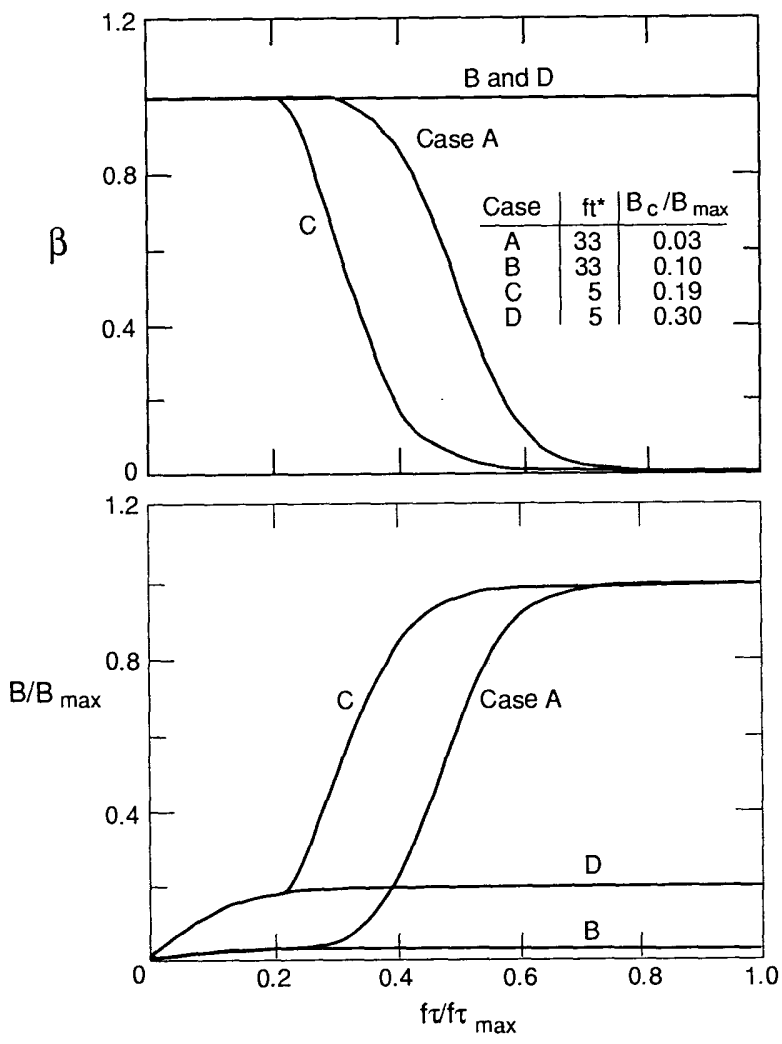

Figure 5 corresponding to these empirical criteria all represent well-mixed frazil. In the model, equilibrium profiles vary continuously with dimensionless renewal frequency. However, for $\beta(\tau)<1$ the equilibrium profiles are not sustainable and the flow interior is progressively depleted of crystals.

Gosink and Osterkamp (1983) reported field data on the Chatanika River and Goldstream Creek in Alaska. They observed well-mixed frazil in the former and layered frazil in the latter. Using the mean depth and the shear velocity values reported, and again taking constant $V=0.01 \mathrm{~m} / \mathrm{s}$, the dimensionless renewal frequency is 33 for Chatanika and 25 for Goldstream. Both values represent modeled equilibrium concentration profiles that are well mixed. The discrepancy between these results and the Goldstream Creek observations may be caused by ice on the water surface that was not drifting freely and a near-surface velocity that was much slower than the maximum of the profile. Since the equation for renewal frequency assumes a logarithmic velocity distribution, the estimate of $f t^{*}$ may not be valid.

Based on the renewal frequency relationship and the assumed constant rise velocity, the empirical relationships and field observations discussed above indicate $f t^{*} \approx 20$ as representative of the flow conditions at the threshold between mixed and layered frazil. The model provides no means to determine the appropriate value of $B_{\mathrm{c}}$ or $B_{\mathrm{c}} / B_{\max }$, which must come from laboratory and field measurements. We are not aware of available measurements that would provide this information. Using Figure 6 we obtain $B_{\mathrm{c}} / B_{\max } \approx 0.05$ for the model to reproduce the observations of mixed or layered frazil. This result indicates that only a few percent of the total mass of frazil is needed at the water surface to initiate bonding between crystals.

We will now apply the model to the Chatanika River case discussed above with $f t^{*}$ and $B_{\mathrm{c}} / B_{\max }$ taken as constants. The initial mass of frazil is assumed to be negligibly small, and the heat loss from the river is assumed to be constant at $10 \mathrm{~W} / \mathrm{m}^{2}$. The development of structure in the surface layer depends on $B_{\mathrm{d}} / B_{\max }$. With $f t^{*}=33$, layered frazil develops for $B_{\mathrm{c}} / B_{\max }=0.03$, and the frazil remains well mixed for $B_{\mathrm{c}} / B_{\max }=0.10$. These results, expressed as $\beta$ and $B / B_{\max }$ with time, are presented in Figure 9 . The 

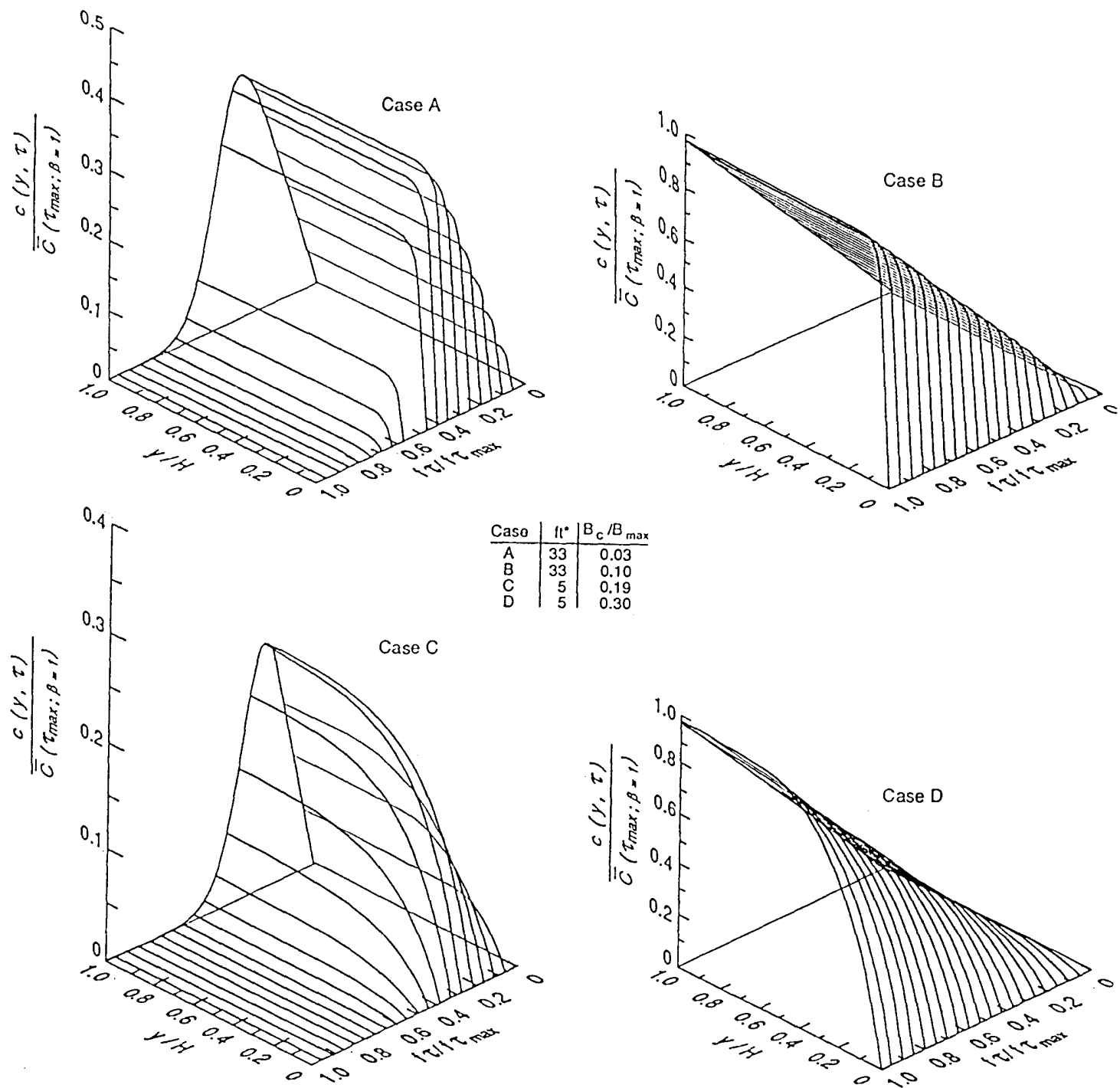

Figure 10. Normalized frazil concentration as a function of dimensionless depth and normalized dimensionless time for selected values of $\mathrm{ft}^{*}$ and $\mathrm{B}_{c} / \mathrm{B}_{\text {max }}$. Note the differences in the concentration scales.

dimensionless time in this figure $f \tau$ is normalized by $f \tau_{\max }$ of 20 . The equilibrium surface layer thickness is only a few percent of $B_{\max }$. Next, we reduce the shear velocity, corresponding to a decrease in the intensity of the turbulence, and obtain $f t^{*}=5$. Layered frazil now develops at $B_{\mathrm{c}} / B_{\max }=0.19$, and the frazil is well mixed with $B_{\mathrm{c}} / B_{\max }=0.30$ (Fig. 9). The dimensional time represented in Figure 9 is much greater at a given normalized dimensionless time for the smaller value of $f t^{*}$. In both cases that developed layered frazil, the surface layer approached equilibrium until disrupted with $\beta<1$.

A set of three-dimensional plots of normalized frazil concentration in depth and time are presented in Figure 10 for these same cases. The parameter $\bar{C}\left(\tau_{\max } ; \beta=1\right)$ used to normalize the concentration is the mean volumetric concentration of frazil in the water column at $\tau_{\max }$ if we assume that $\beta=1$. For $f t^{*}=33$ the concentration profiles at early times are vertically well-mixed and normalized concentrations gradually increase. Subsequently, the collapse or continued growth of the profile depends on $B_{\mathrm{c}} / B_{\max }$. At $f t^{*}=5$ the normalized concentration is not uniform in the vertical direction, but all other trends are the same. 


\section{CONCLUSIONS}

A model is presented for the evolution of frazil with time and over depth in a turbulent flow. The mechanism in the model for counteracting the buoyancy of the crystals is that large energy-containing eddies well up to the surface and entrain all or a portion of the frazil at the surface back into the interior. The crystals in circulation are assumed to be uniformly distributed over the depth after large eddy mixing. The crystals then resume upward migration at their turbulent rise velocity until interrupted by the next renewal eddy. An assumed probability density function is used to obtain averages for parameters over an ensemble of discrete local volumes including concentration profile, surface age, and surface layer thickness.

Starting from a well-mixed initial state with seed-size crystals, a surface layer of frazil develops with time. The thickness of this layer depends on the time and rate of heat loss through the water surface, the frazil concentration of the surface layer that naturally evolves, and the dimensionless renewal frequency. This frequency is the product of the frequency of surface renewal and the gathering period of frazil to the surface. The gathering period depends on the rise velocity, which increases with time according to the rate of heat loss. The dimensionless renewal frequency also characterizes the frazil distribution at equilibrium as well-mixed for $f t^{*}>>1$, or buoyancy dominated and layered for $f t^{*}<1$. The dimensionless renewal frequency and a critical surface layer thickness determine whether the surface layer develops structure. Depending on the development of structure in the surface layer, the frazil will tend toward either an equilibrium profile and surface layer thickness, or a zero concentration profile with all the frazil layered at the surface. Existing empirical criteria and field data on the transition between layered and well-mixed frazil in rivers provide a consistent surface renewal frequency at the transition and an initial estimate of the critical surface layer thickness.

This model for the vertical distribution of frazil in a water column contains parameters that quantify several critical processes in the evolution of frazil from crystals to larger ice forms. We have used the model to systematically assess observations of mixed and layered frazil, and more detailed analyses could be performed. At present the model is a useful tool that can be used to guide future observations of frazil evolution. Additional measurements that characterize the surface renewal frequency, the turbulent rise velocity of frazil, and the critical surface layer thickness are needed to refine and provide further validation of this model.

\section{LITERATURE CITED}

Arakawa, K. (1954) Studies on the freezing of water. II. Formation of disc crystals. Journal of the Faculty of Science, Hokkaido University, Series II, 5, p. 310-339.

Ashton, G.D. (Ed.) (1986) River and Lake Ice Engineering. Littleton, Colorado: Water Resources Publications.

Brown, L.C. (1970) Oxygen transfer in open channel flow. Ph.D. Thesis, University of Wisconsin. Brutsaert, W. and G.H. Jirka (Ed.) (1984) Gas Transfer at Water Surfaces. Dordrecht: Reidel.

Carstens, T. (1966) Experiments with supercooling and ice formation in flowing water. Geofysiske Publikasjoner, 26(9): 1-18.

Daly, S.F. (1984) Frazil ice dynamics. USA Cold Regions Research and Engineering Laboratory, Monograph 84-1.

Daly, S.F. and S.C. Colbeck (1986) Frazil ice measurements in CRREL's flume facility. In Proceedings of the IAHR Symposium on Ice, vol. 1, p. 427-438.

Dankwerts, P.V. (1951) Significance of liquid film coefficients in gas absorption. Industrial al: Engineering Chemistry, 43: 1460-1467.

Davis, J.T. and F.J. Lozano (1979) Turbulence characteristics and mass transfer at air . surfaces. American Institute of Chemical Engineers Journal, 25: 405-415. 
Gordon,C.M. and C.F. Dohne (1973) Some observations of turbulent flow in a tidal estuary. Journal of Geophysical Research, 78: 1971-1978.

Gosink, J.P. and T.E. Osterkamp (1983) Measurements and analysis of velocity profiles and frazil ice-crystal rise velocities during periods of frazil ice formation in rivers. Annals of Glaciology, 4: 79-84.

Hirayama, K. (1986) Growth of ice cover in steep and small rivers. In Proceedings of the IAHR Ice Symposium, Iowa City, Iowa, p. 451-464.

Hobbs, P.V. (1974) Ice Physics. Oxford: Oxford University Press.

Kivisild, H.R. (1970) River and lake ice terminology. International Association for Hydraulic Research paper $1.0,14 \mathrm{p}$.

Komori, S., H. Ueda, F. Ogino and T. Mizushina (1982) Turbulence structure and transport mechanism at the free surface in an open channel flow. International Journal of Heat and Mass Transfer, 25(4): 513-521.

Martin, S. (1981) Frazil ice in rivers and oceans. Annual Review of Fluid Mechanics, 13: 379-397.

Martin, S. and P. Kauffman (1981) A field and laboratory study of wave damping by grease ice. Journal of Glaciology, 27(96): 283-313.

Matousek, V. (1984) Types of ice run and conditions for their formation. In Proceedings of the IAHR Symposium on Ice, Hamburg, Germany, p. 315-327.

Michel, B. (1978) Ice Mechanics. Quebec: Les Presses de L'Universite Laval.

O'Connor, D.J. and W.D. Dobbins (1958) Mechanism of reaeration in natural streams. Transactions of American Society of Civil Engineers, 123: 641-666.

Omstedt, A. (1985) On supercooling and ice formation in turbulent sea-water. Journal of Glaciology, 31(109): 263-271.

Osterkamp, T.E. (1978) Frazil ice formation: A review. Journal of the Hydraulics Division, Proceedings of the American Society of Civil Engineers, 104(Hy9): 1239-1255.

Osterkamp, T.E. and J.P. Gosink (1982) Selected aspects of frazil ice formation and ice cover in turbulent streams. In Proceedings of the Workshop on Hydraulics of Ice-Covered Rivers, June, Edmonton, Alberta, p. 131-147, National Research Council of Canada.

Osterkamp, T.E., R.E. Gilfilian, J.P. Gosink and C.S. Benson (1983) Water temperature measurements in turbulent streams during periods of frazil-ice formation. Annals of Glaciology, International Glaciological Society, Proceedings of the Second Symposium on Applied Glaciology, vol. 4, p. 209-215.

Schaefer, V.J. (1950) The formation of frazil and anchor ice in cold water. Transactions, American Geophysical Union, 31(6): 885-893.

Seitz, R.C. (1973) Observations of intermediate and small-scale turbulent water motion in a stratified tidal estuary (Parts 1 and 2). Chesapeake Bay Institute, Johns Hopkins University, Technical Report 79.

Shimko, M.A. (1985) Scalar transport at a turbulent liquid free surface. M.S. Thesis, Department of Civil Engineering, Massachusetts Institute of Technology.

Sonin, A.A., M.A.Shimko and J.H. Chun (1986) Vapor Condensation onto a turbulent liquid. 1. The steady condensation rate as a function of liquid side turbulence. International Journal of Heat and Mass Transfer, 29(9): 1319-1332.

Tsang, G. (1988) A theory for frazil distribution in turbulent flow. In Proceedings of the IAHR Ice Symposium, Sapporo, Japan, p. 152-169.

Wuebben, J.L. (1984) The rise pattern and velocity of frazil ice. In Proceedings, Workshop on Hydraulics of River Ice, University of New Brunswick, June 20-21, p. 297-316. 


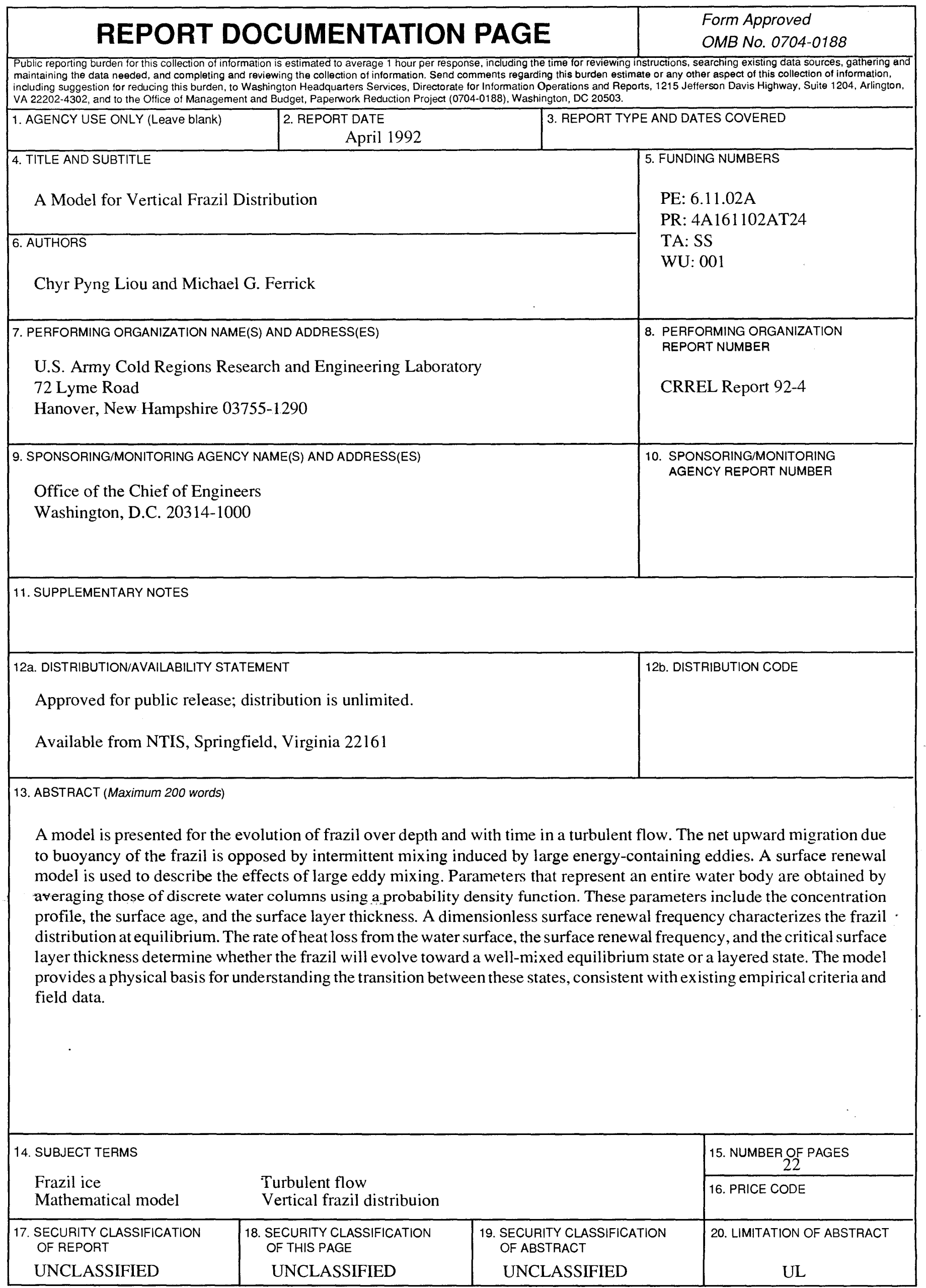

